Canadian

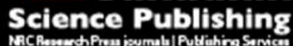

Applied Physiology, Nutrition, and Metabolism Physiologie appliquée, nutrition et métabolisme

\title{
Early metabolic response after resistance exercise with blood flow restriction in well-trained men: a metabolomics approach
}

\begin{tabular}{|c|c|}
\hline Journal: & Applied Physiology, Nutrition, and Metabolism \\
\hline Manuscript ID & apnm-2017-0471.R1 \\
\hline Manuscript Type: & Article \\
\hline Date Submitted by the Author: & 20-Sep-2017 \\
\hline Complete List of Authors: & $\begin{array}{l}\text { Valério, Denis; Universidade Estadual de Campinas } \\
\text { Berton, Ricardo; University of Campinas, School of Physical Education } \\
\text { Conceição, Miguel; University of Campinas, School of Physical Education } \\
\text { Canevarolo, Rafael; Molecular Biology Laboratory, Boldrini Children } \\
\text { Hospital } \\
\text { Chacon-Mikahil, Mara Patrícia; University of Campinas, School of Physical } \\
\text { Education } \\
\text { Cavaglieri, Cláudia; University of Campinas, School of Physical Education } \\
\text { Meirelles, Gabriela; Brazilian Center for Research in Energy and Materials, } \\
\text { Brazilian Biosciences National Laboratory } \\
\text { Zeri, Ana; Brazilian Center for Research in Energy and Materials, Brazilian } \\
\text { Synchrotron Light Laboratory - LNLS } \\
\text { Libardi, Cleiton; Universidade Federal de Sao Carlos }\end{array}$ \\
\hline \multicolumn{2}{|l|}{$\begin{array}{r}\text { Is the invited manuscript for } \\
\text { consideration in a Special } \\
\text { Issue? : }\end{array}$} \\
\hline Keyword: & $\begin{array}{l}\text { metabolism, metabolites, kaatsu training, nuclear magnetic resonance, } \\
\text { resistance training < exercise }\end{array}$ \\
\hline
\end{tabular}




\section{Early metabolic response after resistance exercise with blood flow restriction in} well-trained men: a metabolomics approach

Denis F. Valério ${ }^{1}$, Ricardo Berton ${ }^{1}$, Miguel S. Conceição ${ }^{1}$, Rafael R. Canevarolo ${ }^{3,4}$, Mara Patrícia T. Chacon-Mikahil ${ }^{1}$, Cláudia R. Cavaglieri ${ }^{1}$, Gabriela V. Meirelles ${ }^{4}$, Ana C. Zeri ${ }^{5}$, Cleiton A. Libardi ${ }^{1,2^{*}}$.

${ }^{1}$ Laboratory of Exercise Physiology, Faculty of Physical Education, University of Campinas, Campinas, São Paulo, Brazil.

${ }^{2}$ MUSCULAB - Laboratory of Neuromuscular Adaptations to Resistance Training, Department of Physical Education, Federal University of São Carlos, São Carlos, São Paulo, Brazil.

${ }^{3}$ Molecular Biology Laboratory, Boldrini Children Hospital, Campinas, São Paulo, Brazil.

${ }^{4}$ Brazilian Center for Research in Energy and Materials, Brazilian Biosciences National Laboratory, Campinas, São Paulo, Brazil.

${ }^{5}$ Brazilian Center for Research in Energy and Materials, Brazilian Synchrotron Light Laboratory - LNLS, Campinas, São Paulo, Brazil.

\section{* Corresponding Author}

Cleiton Augusto Libardi, Ph. D.

MUSCULAB - Laboratory of Neuromuscular Adaptations to Resistance Training / Department of Physical Education / Federal University of São Carlos - UFSCar Rod. Washington Luiz, km 235 - SP 310, CEP 13565-905, São Carlos, SP, Brazil Phone: +55 16 3351-8767/E-mail: c.libardi@ufscar.br 


\begin{abstract}
The present study aimed to compare the early metabolic response between high-load resistance exercise (HL-RE) and low-load resistance exercise with blood flow restriction (LL-BFR). Nine young well-trained men participated in a randomized crossover design in which each subject completed LL-BFR, HL-RE or condition control (no exercise) with a one-week interval between them. Blood samples were taken immediately before and five minutes after the exercise sessions. Nuclear magnetic resonance (NMR) spectroscopy identified and quantified 48 metabolites, six of which presented significant changes among the exercise protocols. The HL-RE promoted a higher increase in pyruvate, lactate and alanine compared to the LL-BFR and the control. HL-RE and LL-BFR promoted a higher increase in succinate compared to the control, however, there was no difference between HL-RE and LL-BFR. Also, while there was no difference in acetoacetate between HL-RE and LL-BFR, a greater decrease was observed in both compared to the control. Finally, LL-BFR promoted a greater decrease in choline compared to the control. In conclusion, this study provides by metabolomics a new insight in metabolic response between LL-BFR and HL-RE by demonstrating a distinct response to some metabolites that are not commonly analyzed.
\end{abstract}

Keywords: Metabolism, resistance training, kaatsu training, nuclear magnetic resonance, metabolites. 


\section{Introduction}

Metabolomics is a method aiming to identify and quantify a large number of metabolites present in a given biological sample of interest (Fiehn et al. 2000). Additionally, metabolomics relies on an untargeted approach (i.e., its capacity to detect metabolites without previous target setting); thus it could possibly lead to novel biomarkers detection (Newman and Verdin 2014; Ra et al. 2014). In turn, metabolomics might generate new hypotheses on metabolic responses in several science areas (Madrid-Gambin et al. 2017; Zampieri et al. 2017). Specifically, metabolomics has gained prominence in scientific research regarding resistance exercise (RE)-induced metabolic response (Berton et al. 2017; Yde et al. 2013).

In a recent study of our group, we applied the metabolomics method to analyze the acute metabolic response after high-load RE (HL-RE, 70-85\% one repetition maximum [1-RM]). We identified 49 metabolites, of which 13 presented significant alterations following HL-RE. The study highlighted the analyses of systemic metabolites not commonly observed in HL-RE studies, such as 2-oxoisocaproate, 2-hydroxybutyrate, 3hydroxyisobutyrate, lysine, hypoxanthine and pyruvate; therefore demonstrating the potential of metabolomics for new discoveries (Berton et al. 2017).

An alternate approach to HL-RE is the combination of low-load resistance exercise with blood flow restriction (LL-BFR). In fact, LL-BFR has been shown to induce increases in muscle mass in magnitudes comparable to HL-RE, but applying only 2030\% 1-RM (Laurentino et al. 2012; Libardi et al. 2015; Lixandrao et al. 2015; Vechin et al. 2015). Although muscle adaptations induced by LL-BFR have been extensively investigated in the last decades (Ellefsen et al. 2015; Fujita et al. 2007; Loenneke et al. 2010; Takarada et al. 2000), little is known about the metabolic response in response to it. Importantly, the acute metabolites response may produce anabolic effects, 
contributing to muscle growth (Dankel et al. 2017; Loenneke et al. 2011; Schoenfeld 2013). However, previous studies have limited the metabolic response analyses, i.e., they analysed only few metabolites, mainly lactate and inorganic phosphate) (Manini et al. 2012; Poton and Polito 2016; Reeves et al. 2006; Suga et al. 2012; Takarada et al. 2000). Thus, the employment of exploratory methods that allow identification of a large number of metabolites post-RE is essential to understand the broader metabolic response after LL-BFR.

The aim of the present study was to use the metabolomics method to characterize the acute (i.e., pre to five minutes post-RE) metabolic responses promoted by LL-BFR practice, and compare with the traditional HL-RE-induced metabolic outcomes.

\section{Methods}

\section{Participants}

Nine young men well-trained (age $26.4 \pm 4.4$ years, height $172 \pm 0.05 \mathrm{~cm}$, weight $78.0 \pm 7.8 \mathrm{~kg}$, resistance training experience $8.7 \pm 2.1$ years) participated in this study. The inclusion criteria were: $i$ ) all had been resistance training of lower limbs for a minimum of 2 days-per-week for at least two years; ii) did not have musculoskeletal injuries in the lower limbs and iii) had not taken anabolic steroids, at least six months before of the study. Additionally, all participants were informed about possible risk and discomfort of the study and signed an informed consent document. The protocol and consent were approved by Ethics and Research Committee of the University and complied with all ethical standards for research involving human participants set by the Declaration of Helsinki. 


\section{Experimental design}

Initially, the participants were familiarized with the resistance exercises (LL-BFR and HL-RE) and one repetition maximum test (1-RM) on the $45^{\circ}$ leg press machine. The 1-RM test was performed $72 \mathrm{~h}$ after the last familiarizations session. Before each experimental trial, participants were instructed to refrain from exercise training and vigorous physical activity, and from alcohol and caffeine consumption for a minimum of $48 \mathrm{~h}$. In addition, the participants filled out a diet recall of all food and drink intake 24 hours before each experimental trial. Participants performed three experimental trials in a randomized crossover design: i) high-load resistance exercise (HL-RE); ii) resistance exercise with blood flow restriction (LL-BFR) and iii) control condition (CON, when no exercise was performed).

Experimental trials were separated by a one-week recovery period during which time participants maintained their habitual diet and physical activity patterns. Blood samples were collected immediately before (Pre) and five minutes after (Post) each trial.

\section{Maximum dynamic strength test}

The maximum dynamic strength was assessed by $1-\mathrm{RM}$ test, on $45^{\circ}$ leg press exercise. All participants performed general warm-up on a cycle ergometer at $20 \mathrm{~km} \cdot \mathrm{h}$ 1 for 5 minutes, followed by specific warm-up; one set of eight repetitions with $50 \%$ of an estimated 1-RM load and one minute later, one set of three repetitions with $70 \%$ of the estimated 1-RM. After a three minutes rest interval, the test was performed with the aim to obtain the maximum amount of weight $(\mathrm{kg})$ in a complete cycle (flexionextension of the knee joint with total range of motion of $90^{\circ}$ ). The total number of attempts to determine the value of 1-RM was not greater than five. The interval between attempts was set at three minutes (Brown and Weir 2001). 


\section{Dietary intake}

Before each experimental trial, participants were provided with standardized breakfast that consisted of 290 kilocalories (kcal), 60\% carbohydrates (CHO), 25\% FAT and $15 \%$ proteins (PRO). After consuming the standardized prepacked meals, they remain resting for an hour, after the rest were taken the blood samples and protocols HL-RE, LL-BFR or CON. Total calories (kcal) and proportions of CHO, PRO and FAT were quantified by Dietpro 5.1 software. Water and food intake were not allowed until the end of the experiment.

\section{High-load resistance exercise (HL-RE)}

HL-RE was composed of 3 sets of 10 repetitions $45^{\circ}$ leg press exercise at $80 \% 1$ RM. Each set was separated by a 1 minute recovery period during which time participants remained seated on the $45^{\circ}$ leg press. Complete concentric/eccentric movements were performed with $90^{\circ}$ of range of motion and strong verbal encouragement was provided during each set. The exercise protocol was based on the position stand for muscle hypertrophy (ACSM 2009).

\section{Low-load resistance exercise with blood flow restriction (LL-BFR)}

LL-BFR was composed of 3 sets of 15 repetitions at 20\% 1-RM, with a cuff strapped over the thigh. All participants completed every repetition from each respective set. An 18-cm wide cuff was placed on the proximal portion of the thigh (inguinal fold region) over the tibial artery and once in position, was inflated until an absence of auditory blood pulse detected through auscultation with a vascular Doppler probe (DV600; Marted). Pressure was then slowly released until the first arterial pulse was detected which was considered the systolic pressure at the tibial artery. Cuff pressure 
was set at $80 \%$ of the maximum tibial arterial pressure and the cuff was inflated throughout the entire exercise session (Laurentino et al. 2012). This exercise protocol was chosen due to similar muscle hypertrophy to HL-RE (Laurentino et al. 2012; Libardi et al. 2015; Lixandrao et al. 2015; Vechin et al. 2015).

\section{Control condition (CON)}

The participants of the CON did not perform any exercise. After consuming the standardized breakfast and remaining at rest for an hour at rest, blood collections were performed Pre and Post (5 minutes) rest.

\section{Blood Collection}

Blood samples were collected from the vein of the antecubital fossa. Approximately eight milliliters of blood samples were taken for each time point (Pre and Post exercise) and let clot in vacutainer tubes at room temperature for 30 minutes. The clot blood samples were then centrifuged at $1 \times 10^{3} \mathrm{~g}$ for 15 minutes and the resulting serum was stored at $-80^{\circ} \mathrm{C}$ for subsequent analysis.

\section{Sample preparation for nuclear magnetic resonance (NMR) analysis}

Sample preparation was performed following descriptions previously published (Berton et al. 2017). Briefly, two hundred microliters of filtered serum (filtered through Amicon Ultra 3k $0.5 \mathrm{~mL}$ Centrifugal Filters, EMD Millipore) were reconstituted to 600 $\mu \mathrm{L}$ containing $10 \%$ deuterium oxide $\left(\mathrm{D}_{2} \mathrm{O}, 99.9 \%\right.$; Cambridge Isotope Laboratories Inc., Tewksbury, MA, USA) for locking, $0.1 \mathrm{~mol} \cdot \mathrm{L}-1$ phosphate buffer (stock solution at 1 $\mathrm{mol} \cdot \mathrm{L}-1$ containing $\mathrm{NaH}_{2} \mathrm{PO}_{4}$ at $2.8 \times 10^{-1} \mathrm{~mol} \cdot \mathrm{L}-1$ and $\mathrm{Na}_{2} \mathrm{HPO}_{4}$ at $7.2 \times 10^{-1} \mathrm{~mol} \cdot \mathrm{L}-1$, $\mathrm{pH}=7.4$ ) for $\mathrm{pH}$ standardization and $0.5 \mathrm{mM}$ TSP (3-(Trimethylsilyl) propanoic acid; 
Sigma-Aldrich) for internal reference. Samples were added into $5 \mathrm{~mm}$ proton nuclear magnetic resonance $\left({ }^{1} \mathrm{H}-\mathrm{NMR}\right)$ tubes for immediate acquisition. For more details, see an informative work on metabolomics procedures for NMR spectroscopy of biofluids (Beckonert et al. 2007).

\section{NMR data acquisition and metabolite identification}

${ }^{1} \mathrm{H}-\mathrm{NMR}$ spectra of samples were acquired using a Agilent Inova NMR spectrometer (Agilent Technologies Inc., Santa Clara, CA, USA) equipped with a $5 \mathrm{~mm}$ triple resonance cold probe and operating at a ${ }^{1} \mathrm{H}$ resonance frequency of $600 \mathrm{MHz}$ and constant temperature of $298 \mathrm{~K}\left(25^{\circ} \mathrm{C}\right)$. A total of 256 free induction decays (FID) were collected with $32 \mathrm{~K}$ data points over a spectral width of $16 \mathrm{ppm}$. A $1.5 \mathrm{~s}$ relaxation delay was incorporated between FIDs, during which a continual water pre-saturation radio frequency (RF) field was applied. Spectral phase, baseline corrections, and metabolites identification and quantification were performed with Chenomx NMR Suite 7.6 software (Chenomx Inc., Edmonton, Canada). The spectral region from 4.70 to 5.50 ppm was excluded to remove variability due to suppression of the water resonance signal. To avoid any bias, samples were randomly profiled.

\section{Statistical analysis}

Initially, was performed for each metabolite, Shapiro-Wilk test to verify data normality. In addition, when appropriate, raw data were log transformed to obtain normality. Posteriorly, differences in metabolites concentration along conditions (HLRE, LL-BFR and CON) were accessed through fold change between Post and Pre exercise. To compare fold change of the metabolites and dietary intake (Kcal, CHO, PRO and FAT) between-protocols, a one-way analysis of variance (ANOVA) was 
implemented. In case of significant $F$-values a Tukey adjustment was used for multiple comparison purposes. Data were analyzed using the SAS 9.3 statistical package (SAS Institute Inc., Cary, NC, USA). The significance level was set at $P<0.05$.

\section{Results}

No significant difference were detected among HL-RE, LL-RE and CON in the intake dietary (Table 1) one day before exercises (Kcal, $P=0.828$; $\mathrm{CHO}, P=0.876$; PRO, $P=0.984$; and FAT, $P=0.345)$.

\section{- PLEASE INSERT TABLE 1 HERE -}

After experimental trial LL-BFR, HL-RE and CON, ${ }^{1} \mathrm{H}-\mathrm{NMR}$ spectroscopy was able to identify and quantify 48 metabolites, ranging from amino acids to alcohols, carbohydrates to purine derivatives, sulfones to keto acids (Table 2). Due to problems during spectrum acquisition, analyzes were performed on eight participants in the LLBFR and HL-RE and six in the CON.

Only six metabolites presented different concentrations after LL-BFR and HL-RE as compared with CON (Figure 1 and Table 3). Changes in pyruvate concentration after HL-RE (4.97-fold) were significantly greater that in LL-BFR (3.14-fold; $P=0.0003$ ) and CON (1.34-fold; $P=0.0001)$, respectively). Additionally, changes in pyruvate concentration after LL-BFR were significantly greater than in CON $(P=0.0009)$. Regarding lactate, the changes in concentration after HL-RE (6.85-fold) were greater than in LL-BFR (3.16-fold; $P=0.0002)$ and CON (1.11-fold; $P=0.0001)$, as well as, in LL-BFR compared to $\mathrm{CON}(P=0.0450)$. The changes in alanine concentration after HL-RE (1.34-fold) were also significantly greater than in LL-BFR (1.14-fold; $P=$ 
$0.044)$ and $\mathrm{CON}(1.00$-fold; $P=0.001)$, however, there was no significant difference in the changes of alanine concentration between LL-BFR and CON $(P=0.2200)$. Succinate concentration was significantly greater after HL-RE (3.77-fold) and LL-BFR (2.32-fold) compared to CON (1.09-fold; $P=0.0202$ and $P=0.0089$, respectively), however, there was no significant difference in the change of Succinate concentration between HL-RE and LL-BFR $(P=0.1092)$. The HL-RE $(0.58$-fold $)$ and LL-BFR $(0.75-$ fold) showed significant decrease in the acetoacetate concentration compared to CON (1.36-fold; $P=0.0002, P=0.0019$, respectively), however, there was no significant difference in the changes of acetoacetate concentration between HL-RE and LL-BFR ( $P$ $=0.470)$. LL-BFR protocol showed a significant decrease in the choline concentration (0.89-fold) compared to CON (1.16-Fold; $P=0.0309)$, but no significant difference was observed in the changes of choline concentration between HL-RE and LL-BFR (0.96fold vs. 0.89 -fold; $P=0.7350$ ), as well as, between HL-RE and CON (1.16-fold; $P=$ $0.1230)$.

- PLEASE INSERT TABLE 2 HERE -

- PLEASE INSERT FIGURE 1 HERE -

- PLEASE INSERT TABLE 3 HERE -

\section{Discussion}

The present study aimed to compare the early acute metabolic response after LLBFR and HL-RE using metabolomics in well-trained men. After identifying and quantifying 48 metabolites, we was observed that: i) HL-RE promoted a higher increase 
in pyruvate, lactate and alanine compared with LL-BFR and CON; ii) HL-RE and LLBFR promoted a higher increase in succinate compared with $\mathrm{CON}$, but similar increases between HL-RE and LL-BFR; iii) HL-RE and LL-BFR produced a higher decrease in acetoacetate compared with CON, with no difference between HL-RE and LL-BFR; and iv) LL-BFR promoted a higher decrease in choline compared to CON, but no difference between LL-BFR and HL-RE. Thus, due to the untargeted approach, the use of the metabolomics allowed unprecedented observation and comparison of systemic alterations in alanine, succinate, acetoacetate and choline between LL-BFR and HL-RE.

Pyruvate and lactate concentrations increased significantly following both RE protocols; however, HL-RE promoted increases in those metabolites in a greater extent than did LL-BFR. Thus, we suggest a greater reliance in the anaerobic metabolism (i.e., greater energetic demand) (Medbo and Tabata 1993) in HL-RE compared with LLBFR, at least during initial (i.e., five minutes) post-RE recovery. In contrast, past studies using trained individuals observed similar increase in pyruvate and lactate between LLBFR and HL-RE (Loenneke et al. 2016; Reeves et al. 2006). Although the exact reason for disagreement cannot be ascertained, the difference could rely on the RE protocols applied. While the abovementioned studies used 70\% 1-RM for HL-RE and 20-30\% 1RM for LL-BFR (Loenneke et al. 2016; Reeves et al. 2006), we applied 80\% 1-RM for HL-RE and 20\% 1-RM for LL-BFR. Possibly the larger RE intensity difference between HL-RE and LL-BFR (i.e., $80 \%-20 \%=60 \%$ difference) in our study, might be a reason for the disagreement with previous reports. Additionally, the cuff width, number of repetitions and number of sets could also have contributed to difference between studies.

Alanine concentration was significantly increased after HL-RE compared with both LL-BFR and CON. The increased production of alanine following HL-RE may be 
related to a defense mechanism and gluconeogenesis. During short duration and high load exercise, there is an increase in muscle ammonia production by deamination of adenosine monophosphate and branched chain amino acids (Graham et al. 1997). Furthermore, the removal of muscle ammonia (toxic to humans) is carried out through alanine, which is synthesized from pyruvate transamination derived from glycolysis break. Alanine is carried by the bloodstream to the liver, where it is converted into glucose (glucose-alanine cycle) and ammonia is converted into urea for subsequent elimination. Additionally and corroborating with our results, due to the higher availability of pyruvate after HL-RE, there is a greater capacity to alanine carry ammonia (Felig and Wahren 1971).

The participation of aerobic metabolism was also observed through the succinate metabolite (intermediate metabolite of the tricarboxylic acid cycle [TCA]) (Wells et al. 2009). Our results showed similar increases in succinate concentration after LL-BFR and HL-RE, which were greater than CON. Notably, the similar response between LLBFR and HL-RE is novel. Glycolytic system recovery is performed by the aerobic metabolism, specifically by oxidation of lactate and fatty acids (Scott 2011). Thus, due to a higher lactate production after HL-RE than LL-BFR, it was expected higher increases in succinate concentration following HL-RE than LL-BFR. The reason for similar succinate response between RE protocols is uncertain; however, the analysis of only one time point ( 5 minutes) post-RE precludes a global picture of changes over time. Therefore, additional studies - preferably with several collection points after RE (e.g., 5, 10 and 15 minutes) - are commended.

As aforementioned, energy recovery after exercise can be performed by aerobic metabolism through the oxidation of fatty acids. Acetoacetate is derived from betaoxidation of fatty acid in the liver, and subsequently converted into acetyl-CoA and 
used in the TCA (Newman and Verdin 2014). Accordingly, we observed a similar decrease in acetoacetate concentration after both HL-RE and LL-BFR compared with CON. Thus, it is possible to suggest that the aerobic metabolism is activated similarly after both LL-BFR and HL-RE. However, analogously to succinate, this result should be interpreted with caution. As HL-RE showed a higher reliance on the anaerobic metabolism than LL-BFR, we also expected a larger increase in the acetoacetate concentration after HL-RE compared with LL-BFR. Thus, additional studies must be performed with more time points of analyses. In addition, it is important to note that acetoacetate concentration changes following HL-RE were not observed in another study of our group using metabolomics (Berton et al. 2017). The difference may be related to training level of the subjects. While in the present study participants had on average $8.7 \pm 2.1$ years of experience in resistance training, in the study of Berton et al. (2017), the participants were untrained. Corroborating with our suggestion, studies have shown that training status can exert an effect on acetoacetate metabolism (Johnson and Walton 1972; Johnson et al. 1969). Specifically, untrained individuals showed lower utilization rates of this metabolite compared with trained counterparts (Johnson and Walton 1972; Johnson et al. 1969).

Choline was another altered metabolite, depicting a significant decrease following LL-BFR compared to CON, but no difference between LL-BFR and HL-RE. Choline is an important component of the cell membrane and is an acetylcholine precursor (von Allworden et al. 1993). It is likely that choline alteration following LL-BFR is not related to membrane integrity, as this type of exercise does not seem to induce large magnitudes of muscle damage (Loenneke et al. 2014). Additionally, if a large level of muscle damage was present, it would be expected a higher (as vs. lower reported herein) choline concentration in bloodstream compared to CON. Thus, we believe that the 
decrease in choline concentration is related to acetylcholine. As mentioned, choline is a precursor of acetylcholine, an important neurotransmitter of excitation-contraction coupling at the neuromuscular junction (Conlay et al. 1992). Therefore, it is suggested that the decrease in acetylcholine acutely post-RE can cause inhibition of the excitationcontraction coupling and contribute to a decline in muscle performance (Conlay et al. 1992). Although choline is a understood metabolite, we are not aware of the exact reason why the lowering of the choline only occurred in the LL-BFR. Thus, further studies are required to investigate the choline response after LL-BFR.

The limitations of the study may be related to dietary standardization one day before RE protocols and the time points of blood analyses. Despite a standardized breakfast, the diet of the entire day before the exercise can influence the metabolic response. To reduce this interference, participants were asked to maintain their nutritional habits. All participants completed a diet recall regarding the day before performing each RE protocol and the CON day. From this diet recall, we observed that total calories, as well as $\mathrm{CHO}, \mathrm{PRO}$ and FAT were similar in the three days prior to each experimental condition. Although, the early acute response (5 minutes) is important, future studies must perform additional blood sampling/analyses (e.g., 15, 30, 60 minutes) post-exercise for continued knowledge on the global changes in metabolic response throughout recovery.

In conclusion, this study provides by metabolomics new insights regarding the metabolic responses between LL-BFR and HL-RE. We demonstrate that HL-RE promoted a higher increase in pyruvate, lactate and alanine compared to LL-BFR, while similar alterations in succinate and acetoacetate were observed between RE protocols. Additionally, only LL-BFR promoted a decrease in choline concentrations after RE. 


\section{Acknowledgments}

The authors acknowledge the Brazilian Biosciences National Laboratory (LNBio) at the Brazilian Center for Research in Energy and Materials (CNPEM), Campinas, Brazil, for all support with the NMR spectrometer.

\section{Disclosure statement}

No potential conflict of interest was reported by the authors.

\section{References}

American College of Sports, M. 2009. American College of Sports Medicine position stand. Progression models in resistance training for healthy adults. Med. Sci. Sports Exerc. 41 (3):687-708. doi: 10.1249/MSS.0b013e3181915670.

Beckonert, O., Keun, H.C., Ebbels, T.M., Bundy, J., Holmes, E., Lindon, J.C. and Nicholson, J.K. 2007. Metabolic profiling, metabolomic and metabonomic procedures for NMR spectroscopy of urine, plasma, serum and tissue extracts. Nat. Protoc. 2 (11):2692-2703. doi: 10.1038/nprot.2007.376.

Berton, R., Conceição, M.S., Libardi, C.A., Canevarolo, R.R., Gáspari, A.F., ChaconMikahil, M.P., Zeri, A.C. and Cavaglieri, C.R. 2017. Metabolic time-course response after resistance exercise: A metabolomics approach. J. Sports Sci. 35 (12):1211-1218. doi: 10.1080/02640414.2016.1218035.

Brown, L.E. and Weir, J.P. 2001. ASEP Procedures Recommendations I: Accurate Assessment of Muscular Strength and Power. J. Exerc. Physiol. 2001;4(3):1-21. J. Exerc. Physiol. 4 (3):1-21. 
Conlay, L.A., Sabounjian, L.A. and Wurtman, R.J. 1992. Exercise and neuromodulators: choline and acetylcholine in marathon runners. Int. J. Sports Med. 13 Suppl 1:S141-142. doi: 10.1055/s-2007-1024619.

Dankel, S.J., Mattocks, K.T., Jessee, M.B., Buckner, S.L., Mouser, J.G. and Loenneke, J.P. 2017. Do metabolites that are produced during resistance exercise enhance muscle hypertrophy? Eur. J. Appl. Physiol. doi: 10.1007/s00421-017-3690-1.

Ellefsen, S., Hammarstrom, D., Strand, T.A., Zacharoff, E., Whist, J.E., Rauk, I., Nygaard, H., Vegge, G., Hanestadhaugen, M., Wernbom, M., et al. 2015. Blood flow-restricted strength training displays high functional and biological efficacy in women: a within-subject comparison with high-load strength training. Am. J. Physiol. Regul. Integr. Comp. Physiol. 309 (7):R767-779. doi: 10.1152/ajpregu.00497.2014.

Felig, P. and Wahren, J. 1971. Amino acid metabolism in exercising man. J. Clin. Invest. 50 (12):2703-2714. doi: 10.1172/JCI106771.

Fiehn, O., Kopka, J., Dormann, P., Altmann, T., Trethewey, R.N. and Willmitzer, L. 2000. Metabolite profiling for plant functional genomics. Nat. Biotechnol. 18 (11):1157-1161. doi: 10.1038/81137.

Fujita, S., Abe, T., Drummond, M.J., Cadenas, J.G., Dreyer, H.C., Sato, Y., Volpi, E. and Rasmussen, B.B. 2007. Blood flow restriction during low-intensity resistance exercise increases $\mathrm{S} 6 \mathrm{~K} 1$ phosphorylation and muscle protein $\begin{array}{llllll}\text { synthesis. } & \text { J. Appl. Physiol. (1985) } 103 \text { (3):903-910. doi: }\end{array}$ 10.1152/japplphysiol.00195.2007.

Graham, T.E., Turcotte, L.P., Kiens, B. and Richter, E.A. 1997. Effect of endurance training on ammonia and amino acid metabolism in humans. Med. Sci. Sports Exerc. 29 (5):646-653. 
Johnson, R.H. and Walton, J.L. 1972. The effect of exercise upon acetoacetate metabolism in athletes and non-athletes. Q. J. Exp. Physiol. Cogn. Med. Sci. 57 (1):73-79.

Johnson, R.H., Walton, J.L., Krebs, H.A. and Williamson, D.H. 1969. Metabolic fuels during and after severe exercise in athletes and non-athletes. Lancet, 2 (7618):452-455.

Laurentino, G.C., Ugrinowitsch, C., Roschel, H., Aoki, M.S., Soares, A.G., Neves, M., Jr., Aihara, A.Y., Fernandes Ada, R. and Tricoli, V. 2012. Strength training with blood flow restriction diminishes myostatin gene expression. Med. Sci. Sports Exerc. 44 (3):406-412. doi: 10.1249/MSS.0b013e318233b4bc.

Libardi, C.A., Chacon-Mikahil, M.P., Cavaglieri, C.R., Tricoli, V., Roschel, H., Vechin, F.C., Conceicao, M.S. and Ugrinowitsch, C. 2015. Effect of concurrent training with blood flow restriction in the elderly. Int. J. Sports Med. 36 (5):395-399. doi: $10.1055 / \mathrm{s}-0034-1390496$.

Lixandrao, M.E., Ugrinowitsch, C., Laurentino, G., Libardi, C.A., Aihara, A.Y., Cardoso, F.N., Tricoli, V. and Roschel, H. 2015. Effects of exercise intensity and occlusion pressure after 12 weeks of resistance training with blood-flow restriction. Eur. J. Appl. Physiol. 115 (12):2471-2480. doi: 10.1007/s00421015-3253-2.

Loenneke, J.P., Fahs, C.A., Wilson, J.M. and Bemben, M.G. 2011. Blood flow restriction: the metabolite/volume threshold theory. Med. Hypotheses, 77 (5):748-752. doi: 10.1016/j.mehy.2011.07.029.

Loenneke, J.P., Kim, D., Fahs, C.A., Thiebaud, R.S., Abe, T., Larson, R.D., Bemben, D.A. and Bemben, M.G. 2016. The influence of exercise load with and without 
different levels of blood flow restriction on acute changes in muscle thickness and lactate. Clin. Physiol. Funct. Imaging, doi: 10.1111/cpf.12367.

Loenneke, J.P., Thiebaud, R.S. and Abe, T. 2014. Does blood flow restriction result in skeletal muscle damage? A critical review of available evidence. Scand. J. Med. Sci. Sports, 24 (6):e415-422. doi: 10.1111/sms.12210.

Loenneke, J.P., Wilson, G.J. and Wilson, J.M. 2010. A mechanistic approach to blood flow occlusion. Int. J. Sports Med. 31 (1):1-4. doi: 10.1055/s-0029-1239499.

Madrid-Gambin, F., Llorach, R., Vazquez-Fresno, R., Urpi-Sarda, M., AlmanzaAguilera, E., Garcia-Aloy, M., Estruch, R., Corella, D. and Andres-Lacueva, C. 2017. Urinary $1 \mathrm{H}-\mathrm{NMR}$ metabolomic fingerprinting reveals biomarkers of pulse consumption related to energy metabolism modulation in a subcohort from the PREDIMED study. J. Proteome Res. doi: 10.1021/acs.jproteome.6b00860.

Manini, T.M., Yarrow, J.F., Buford, T.W., Clark, B.C., Conover, C.F. and Borst, S.E. 2012. Growth hormone responses to acute resistance exercise with vascular restriction in young and old men. Growth Horm. IGF. Res. 22 (5):167-172. doi: 10.1016/j.ghir.2012.05.002.

Medbo, J.I. and Tabata, I. 1993. Anaerobic energy release in working muscle during 30 s to 3 min of exhausting bicycling. J. Appl. Physiol. (1985) 75 (4):1654-1660.

Newman, J.C. and Verdin, E. 2014. Ketone bodies as signaling metabolites. Trends Endocrinol Metab. 25 (1):42-52. doi: 10.1016/j.tem.2013.09.002.

Poton, R. and Polito, M.D. 2016. Hemodynamic response to resistance exercise with and without blood flow restriction in healthy subjects. Clin. Physiol. Funct. Imaging, 36 (3):231-236. doi: 10.1111/cpf.12218. 
Ra, S.G., Maeda, S., Higashino, R., Imai, T. and Miyakawa, S. 2014. Metabolomics of salivary fatigue markers in soccer players after consecutive games. Appl. Physiol. Nutr. Metab. 39 (10):1120-1126. doi: 10.1139/apnm-2013-0546.

Reeves, G.V., Kraemer, R.R., Hollander, D.B., Clavier, J., Thomas, C., Francois, M. and Castracane, V.D. 2006. Comparison of hormone responses following light resistance exercise with partial vascular occlusion and moderately difficult resistance exercise without occlusion. J. Appl. Physiol. (1985) 101 (6):16161622. doi: 10.1152/japplphysiol.00440.2006.

Schoenfeld, B.J. 2013. Potential mechanisms for a role of metabolic stress in hypertrophic adaptations to resistance training. Sports Med. 43 (3):179-194. doi: 10.1007/s40279-013-0017-1.

Scott, C.B. 2011. Quantifying the immediate recovery energy expenditure of resistance training. J. Strength. Cond. Res. 25 (4):1159-1163. doi: 10.1519/JSC.0b013e3181d64eb5.

Suga, T., Okita, K., Takada, S., Omokawa, M., Kadoguchi, T., Yokota, T., Hirabayashi, K., Takahashi, M., Morita, N., Horiuchi, M., et al. 2012. Effect of multiple set on intramuscular metabolic stress during low-intensity resistance exercise with blood flow restriction. Eur. J. Appl. Physiol. 112 (11):3915-3920. doi: $10.1007 / \mathrm{s} 00421-012-2377-x$.

Takarada, Y., Takazawa, H., Sato, Y., Takebayashi, S., Tanaka, Y. and Ishii, N. 2000. Effects of resistance exercise combined with moderate vascular occlusion on muscular function in humans. J. Appl. Physiol. (1985) 88 (6):2097-2106.

Vechin, F.C., Libardi, C.A., Conceicao, M.S., Damas, F.R., Lixandrao, M.E., Berton, R.P., Tricoli, V.A., Roschel, H.A., Cavaglieri, C.R., Chacon-Mikahil, M.P., et al. 2015. Comparisons between low-intensity resistance training with blood flow 
restriction and high-intensity resistance training on quadriceps muscle mass and strength in elderly. J. Strength. Cond. Res. 29 (4):1071-1076. doi: 10.1519/JSC.0000000000000703.

von Allworden, H.N., Horn, S., Kahl, J. and Feldheim, W. 1993. The influence of lecithin on plasma choline concentrations in triathletes and adolescent runners during exercise. Eur. J. Appl. Physiol. Occup. Physiol. 67 (1):87-91.

Wells, G.D., Selvadurai, H. and Tein, I. 2009. Bioenergetic provision of energy for muscular activity. Paediatr. Respir. Rev. 10 (3):83-90. doi: 10.1016/j.prrv.2009.04.005.

Yde, C.C., Ditlev, D.B., Reitelseder, S. and Bertram, H.C. 2013. Metabonomic Response to Milk Proteins after a Single Bout of Heavy Resistance Exercise Elucidated by ${ }^{1} \mathrm{H}$ Nuclear Magnetic Resonance Spectroscopy. Metabolites, 3:33-46.

Zampieri, M., Sekar, K., Zamboni, N. and Sauer, U. 2017. Frontiers of high-throughput metabolomics. Curr. Opin. Chem. Biol. 36:15-23. doi: 10.1016/j.cbpa.2016.12.006. 
Table 1. Dietary intake

\begin{tabular}{llccc}
\hline Protocol & CHO $(\mathrm{g})$ & FAT $(\mathrm{g})$ & PRO $(\mathrm{g})$ & Kcal \\
\hline HL-RE & $258 \pm 59$ & $59 \pm 26$ & $147 \pm 53$ & $2158 \pm 673$ \\
LL-BFR & $227 \pm 69$ & $45 \pm 20$ & $132 \pm 53$ & $1850 \pm 433$ \\
CON & $237 \pm 72$ & $45 \pm 14$ & $120 \pm 49$ & $1843 \pm 533$ \\
\hline
\end{tabular}

HL-RE, high-load resistance exercise; LL-BFR, low-load resistance exercise with blood flow restriction; $\mathrm{CON}$, control condition; $\mathrm{CHO}$, carbohydrates; $\mathrm{PRO}$, proteins. 
Table 2. All absolute values of metabolites before (Pre) and after (Post) high-load resistance exercise (HL-RE), low-load resistance exercise with blood flow restriction (LL-BFR) and control condition (CON) used to calculate fold change.

\begin{tabular}{|c|c|c|c|c|c|c|}
\hline \multirow{2}{*}{ Metabolities } & \multicolumn{2}{|c|}{ HL-RE } & \multicolumn{2}{|c|}{ LL-BFR } & \multicolumn{2}{|c|}{$\mathrm{CON}$} \\
\hline & Pre & Post & Pre & Post & Pre & Post \\
\hline 2-Aminobutyrate & $0.063 \pm 0.018$ & $0.059 \pm 0.029$ & $0.079 \pm 0.026$ & $0.073 \pm 0.02$ & $0.061 \pm 0.020$ & $0.065 \pm 0.020$ \\
\hline 2-Hydroxybutyrate & $0.099 \pm 0.030$ & $0.108 \pm 0.033$ & $0.119 \pm 0.042$ & $0.132 \pm 0.046$ & $0.101 \pm 0.031$ & $0.109 \pm 0.027$ \\
\hline 2-Hydroxyisovalerate & $0.036 \pm 0.007$ & $0.035 \pm 0.003$ & $0.036 \pm 0.003$ & $0.040 \pm 0.011$ & $0.038 \pm 0.008$ & $0.040 \pm 0.011$ \\
\hline 2-Oxoisocaproate & $0.024 \pm 0.004$ & $0.035 \pm 0.008$ & $0.025 \pm 0.003$ & $0.033 \pm 0.004$ & $0.021 \pm 0.002$ & $0.023 \pm 0.006$ \\
\hline 3-Hydroxybutyrate & $0.163 \pm 0.135$ & $0.170 \pm 0.086$ & $0.191 \pm 0.194$ & $0.176 \pm 0.125$ & $0.147 \pm 0.115$ & $0.134 \pm 0.061$ \\
\hline 3-Hydroxyisobutyrate & $0.065 \pm 0.022$ & $0.061 \pm 0.185$ & $0.068 \pm 0.016$ & $0.067 \pm 0.022$ & $0.054 \pm 0.016$ & $0.058 \pm 0.016$ \\
\hline 3-Methyl-2-oxovalerate & $0.020 \pm 0.003$ & $0.031 \pm 0.005$ & $0.020 \pm 0.003$ & $0.026 \pm 0.004$ & $0.016 \pm 0.004$ & $0.022 \pm 0.011$ \\
\hline Acetate & $0.090 \pm 0.037$ & $0.082 \pm 0.035$ & $0.088 \pm 0.022$ & $0.089 \pm 0.030$ & $0.076 \pm 0.038$ & $0.096 \pm 0.035$ \\
\hline Acetoacetate & $0.124 \pm 0.069$ & $0.067 \pm 0.030$ & $0.145 \pm 0.115$ & $0.095 \pm 0.060$ & $0.116 \pm 0.072$ & $0.123 \pm 0.037$ \\
\hline Acetone & $0.030 \pm 0.007$ & $0.028 \pm 0.006$ & $0.033 \pm 0.014$ & $0.027 \pm 0.009$ & $0.027 \pm 0.011$ & $0.027 \pm 0.011$ \\
\hline Alanine & $1.163 \pm 0.179$ & $1.504 \pm 0.239$ & $1.328 \pm 0.254$ & $1.471 \pm 0.200$ & $1.148 \pm 0.245$ & $1.131 \pm 0.207$ \\
\hline Ascorbate & $0.163 \pm 0.100$ & $0.181 \pm 0.057$ & $0.169 \pm 0.067$ & $0.189 \pm 0.088$ & $0.175 \pm 0.070$ & $0.193 \pm 0.101$ \\
\hline Asparagine & $0.230 \pm 0.045$ & $0.234 \pm 0.042$ & $0.248 \pm 0.053$ & $0.239 \pm 0.049$ & $0.215 \pm 0.048$ & $0.194 \pm 0.036$ \\
\hline Betaine & $0.163 \pm 0.051$ & $0.170 \pm 0.060$ & $0.187 \pm 0.049$ & $0.189 \pm 0.042$ & $0.151 \pm 0.061$ & $0.171 \pm 0.071$ \\
\hline Carnitine & $0.199 \pm 0.030$ & $0.196 \pm 0.032$ & $0.221 \pm 0.032$ & $0.204 \pm 0.026$ & $0.196 \pm 0.017$ & $0.197 \pm 0.017$ \\
\hline Choline & $0.028 \pm 0.005$ & $0.026 \pm 0.006$ & $0.031 \pm 0.007$ & $0.028 \pm 0.006$ & $0.025 \pm 0.006$ & $0.027 \pm 0.008$ \\
\hline Citrate & $0.655 \pm 0.143$ & $0.821 \pm 0.164$ & $0.904 \pm 0.122$ & $0.917 \pm 0.132$ & $0.701 \pm 0.143$ & $0.697 \pm 0.232$ \\
\hline Creatine & $0.077 \pm 0.040$ & $0.066 \pm 0.027$ & $0.075 \pm 0.027$ & $0.070 \pm 0.036$ & $0.066 \pm 0.0310$ & $0.081 \pm 0.025$ \\
\hline Creatinine & $0.347 \pm 0.057$ & $0.373 \pm 0.070$ & $0.378 \pm 0.059$ & $0.379 \pm 0.072$ & $0.325 \pm 0.058$ & $0.332 \pm 0.055$ \\
\hline Cystine & $0.384 \pm 0.147$ & $0.343 \pm 0.131$ & $0.290 \pm 0.115$ & $0.318 \pm 0.095$ & $0.272 \pm 0.095$ & $0.290 \pm 0.117$ \\
\hline Dimethyl sulfone & $0.016 \pm 0.004$ & $0.019 \pm 0.005$ & $0.018 \pm 0.007$ & $0.018 \pm 0.005$ & $0.015 \pm 0.006$ & $0.017 \pm 0.006$ \\
\hline Dimethylamine & $0.003 \pm 0.0006$ & $0.003 \pm 0.0007$ & $0.003 \pm 0.001$ & $0.003 \pm 0.0004$ & $0.003 \pm 0.0005$ & $0.003 \pm 0.0003$ \\
\hline Ethanol & $0.554 \pm 0.215$ & $0.676 \pm 0.243$ & $0.675 \pm 0.309$ & $0.605 \pm 0.230$ & $0.345 \pm 0.109$ & $0.306 \pm 0.093$ \\
\hline Formate & $0.029 \pm 0.003$ & $0.027 \pm 0.002$ & $0.034 \pm 0.006$ & $0.033 \pm 0.006$ & $0.026 \pm 0.002$ & $0.032 \pm 0.007$ \\
\hline Glucose & $26.12 \pm 2.079$ & $25.73 \pm 0.294$ & $27.11 \pm 2.291$ & $27.75 \pm 2.739$ & $23.36 \pm 2.504$ & $26.24 \pm 2.285$ \\
\hline Glutamine & $2.564 \pm 0.297$ & $2.827 \pm 0.415$ & $2.792 \pm 0.421$ & $2.657 \pm 0.172$ & $2.359 \pm 0.252$ & $2.341 \pm 0.289$ \\
\hline Glycerol & $0.541 \pm 0.066$ & $0.659 \pm 0.176$ & $0.554 \pm 0.066$ & $0.688 \pm 0.103$ & $0.526 \pm 0.069$ & $0.545 \pm 0.052$ \\
\hline Glycine & $0.544 \pm 0.082$ & $0.559 \pm 0.091$ & $0.576 \pm 0.115$ & $0.564 \pm 0.068$ & $0.523 \pm 0.073$ & $0.529 \pm 0.072$ \\
\hline Histidine & $0.391 \pm 0.027$ & $0.413 \pm 0.045$ & $0.421 \pm 0.037$ & $0.415 \pm 0.055$ & $0.381 \pm 0.041$ & $0.375 \pm 0.072$ \\
\hline Hypoxanthine & $0.041 \pm 0.011$ & $0.085 \pm 0.041$ & $0.042 \pm 0.016$ & $0.055 \pm 0.009$ & $0.039 \pm 0.009$ & $0.042 \pm 0.014$ \\
\hline Inosine & $0.103 \pm 0.036$ & $0.133 \pm 0.048$ & $0.091 \pm 0.039$ & $0.136 \pm 0.043$ & $0.076 \pm 0.022$ & $0.098 \pm 0.038$ \\
\hline Isoleucine & $0.356 \pm 0.062$ & $0.346 \pm 0.062$ & $0.354 \pm 0.060$ & $0.334 \pm 0.041$ & $0.312 \pm 0.030$ & $0.325 \pm 0.067$ \\
\hline Lactate & $5.036 \pm 1.391$ & $30.90 \pm 7.310$ & $5.322 \pm 0.628$ & $16.71 \pm 2.787$ & $4.900 \pm 0.798$ & $5.036 \pm 1.593$ \\
\hline Leucine & $0.622 \pm 0.104$ & $0.587 \pm 0.111$ & $0.659 \pm 0.117$ & $0.585 \pm 0.102$ & $0.549 \pm 0.079$ & $0.560 \pm 0.081$ \\
\hline Lysine & $0.743 \pm 0.117$ & $0.752 \pm 0.109$ & $0.779 \pm 0.097$ & $0.742 \pm 0.087$ & $0.675 \pm 0.095$ & $0.703 \pm 0.088$ \\
\hline Methanol & $0.096 \pm 0.035$ & $0.104 \pm 0.048$ & $0.112 \pm 0.061$ & $0.099 \pm 0.044$ & $0.096 \pm 0.037$ & $0.083 \pm 0.041$ \\
\hline Methionine & $0.138 \pm 0.028$ & $0.145 \pm 0.022$ & $0.162 \pm 0.039$ & $0.164 \pm 0.029$ & $0.127 \pm 0.048$ & $0.149 \pm 0.028$ \\
\hline Methylsuccinate & $0.021 \pm 0.005$ & $0.020 \pm 0.004$ & $0.021 \pm 0.005$ & $0.023 \pm 0.006$ & $0.019 \pm 0.005$ & $0.019 \pm 0.003$ \\
\hline Ornithine & $0.200 \pm 0.053$ & $0.186 \pm 0.051$ & $0.207 \pm 0.040$ & $0.172 \pm 0.033$ & $0.201 \pm 0.042$ & $0.188 \pm 0.055$ \\
\hline Phenylalanine & $0.332 \pm 0.059$ & $0.331 \pm 0.061$ & $0.357 \pm 0.044$ & $0.336 \pm 0.062$ & $0.314 \pm 0.058$ & $0.318 \pm 0.063$ \\
\hline Proline & $0.841 \pm 0.177$ & $0.811 \pm 0.186$ & $0.871 \pm 0.218$ & $0.823 \pm 0.207$ & $0.711 \pm 0.176$ & $0.744 \pm 0.158$ \\
\hline Propylene glycol & $0.011 \pm 0.005$ & $0.013 \pm 0.009$ & $0.010 \pm 0.002$ & $0.013 \pm 0.007$ & $0.008 \pm 0.001$ & $0.012 \pm 0.004$ \\
\hline Pyruvate & $0.223 \pm 0.058$ & $1.017 \pm 0.233$ & $0.246 \pm 0.036$ & $0.750 \pm 0.180$ & $0.192 \pm 0.028$ & $0.247 \pm 0.049$ \\
\hline Serine & $0.415 \pm 0.052$ & $0.385 \pm 0.062$ & $0.437 \pm 0.039$ & $0.434 \pm 0.055$ & $0.386 \pm 0.035$ & $0.420 \pm 0.059$ \\
\hline Succinate & $0.015 \pm 0.004$ & $0.051 \pm 0.010$ & $0.015 \pm 0.004$ & $0.033 \pm 0.005$ & $0.013 \pm 0.002$ & $0.014 \pm 0.003$ \\
\hline Threonine & $0.536 \pm 0.156$ & $0.577 \pm 0.120$ & $0.630 \pm 0.136$ & $0.638 \pm 0.104$ & $0.581 \pm 0.141$ & $0.600 \pm 0.142$ \\
\hline Tyrosine & $0.414 \pm 0.071$ & $0.418 \pm 0.070$ & $0.470 \pm 0.065$ & $0.442 \pm 0.054$ & $0.418 \pm 0.094$ & $0.428 \pm 0.087$ \\
\hline Valine & $1.146 \pm 0.235$ & $1.097 \pm 0.241$ & $1.153 \pm 0.178$ & $1.103 \pm 0.158$ & $1.025 \pm 0.157$ & $1.046 \pm 0.166$ \\
\hline
\end{tabular}

Values as mean \pm standard deviation. 
Table 3. Fold change in metabolites that do not show significant differences between high-load resistance exercise (HL-RE), low-load resistance exercise with blood flow restriction (LL-BFR) and control condition $(\mathrm{CON})$.

\begin{tabular}{|c|c|c|c|c|}
\hline Metabolites & HL-RE & LL-BFR & $\mathrm{CON}$ & $P$-value \\
\hline 2-Aminobutyrate & $0.89 \pm 0.32$ & $0.92 \pm 0.14$ & $1.10 \pm 0.14$ & 0.2950 \\
\hline 2-Hydroxybutyrate & $1.08 \pm 0.05$ & $1.15 \pm 0.24$ & $1.27 \pm 0.41$ & 0.5562 \\
\hline 2-Hydroxyisovalerate & $1.04 \pm 0.09$ & $1.10 \pm 0.24$ & $1.17 \pm 0.44$ & 0.1447 \\
\hline 2-Oxoisocaproate & $1.44 \pm 0.22$ & $1.36 \pm 0.28$ & $1.16 \pm 0.35$ & 0.1447 \\
\hline 3-Hydroxybutyrate & $1.21 \pm 0.40$ & $1.10 \pm 0.18$ & $1.32 \pm 0.22$ & 0.3788 \\
\hline 3-Hydroxyisobutyrate & $1.04 \pm 0.21$ & $0.98 \pm 0.25$ & $1.12 \pm 0.20$ & 0.4819 \\
\hline 3-Methyl-2-oxovalerate & $1.61 \pm 0.36$ & $1.38 \pm 0.34$ & $1.52 \pm 1.34$ & 0.1732 \\
\hline Acetate & $1.02 \pm 0.37$ & $1.08 \pm 0.32$ & $1.31 \pm 0.27$ & 0.3418 \\
\hline Acetone & $0.94 \pm 0.13$ & $0.88 \pm 0.16$ & $1.12 \pm 0.22$ & 0.0639 \\
\hline Ascorbate & $1.50 \pm 1.10$ & $1.35 \pm 0.93$ & $1.18 \pm 0.56$ & 0.8043 \\
\hline Asparagine & $1.04 \pm 0.21$ & $1.03 \pm 0.30$ & $0.92 \pm 0.31$ & 0.6668 \\
\hline Betaine & $1.15 \pm 0.56$ & $1.02 \pm 0.17$ & $1.06 \pm 0.15$ & 0.6824 \\
\hline Carnitine & $1.01 \pm 0.11$ & $0.95 \pm 0.15$ & $1.04 \pm 0.08$ & 0.4307 \\
\hline Citrate & $1.35 \pm 0.49$ & $0.93 \pm 0.41$ & $1.04 \pm 0.19$ & 0.3326 \\
\hline Creatine & $1.01 \pm 0.20$ & $0.93 \pm 0.27$ & $1.17 \pm 0.14$ & 0.1877 \\
\hline Creatinine & $1.07 \pm 0.13$ & $0.99 \pm 0.14$ & $1.02 \pm 0.08$ & 0.5042 \\
\hline Cystine & $0.88 \pm 0.31$ & $1.43 \pm 1.03$ & $1.16 \pm 0.44$ & 0.2528 \\
\hline Dimethyl sulfone & $1.18 \pm 0.27$ & $1.13 \pm 0.45$ & $1.03 \pm 0.27$ & 0.3231 \\
\hline Dimethylamine & $1.12 \pm 0.19$ & $1.13 \pm 0.33$ & $0.94 \pm 0.10$ & 0.4061 \\
\hline Ethanol & $1.21 \pm 0.46$ & $1.08 \pm 0.57$ & $0.97 \pm 0.08$ & 0.3627 \\
\hline Formate & $0.95 \pm 0.09$ & $1.01 \pm 0.28$ & $1.24 \pm 0.37$ & 0.1719 \\
\hline Glucose & $1.00 \pm 0.09$ & $1.03 \pm 0.15$ & $0.91 \pm 0.45$ & 0.6528 \\
\hline Glutamine & $1.07 \pm 0.09$ & $0.97 \pm 0.17$ & $1.00 \pm 0.09$ & 0.3839 \\
\hline Glycerol & $1.25 \pm 0.36$ & $1.22 \pm 0.18$ & $1.08 \pm 0.24$ & 0.5598 \\
\hline Glycine & $0.99 \pm 0.07$ & $0.98 \pm 0.15$ & $1.02 \pm 0.07$ & 0.6823 \\
\hline Histidine & $1.05 \pm 0.11$ & $1.00 \pm 0.17$ & $1.03 \pm 0.13$ & 0.8037 \\
\hline Hypoxanthine & $2.15 \pm 1.36$ & $1.45 \pm 0.56$ & $1.07 \pm 0.38$ & 0.1473 \\
\hline Inosine & $1.43 \pm 0.38$ & $1.79 \pm 1.22$ & $1.12 \pm 0.18$ & 0.0558 \\
\hline Isoleucine & $0.98 \pm 0.04$ & $0.95 \pm 0.19$ & $1.05 \pm 0.17$ & 0.4756 \\
\hline Leucine & $0.96 \pm 0.11$ & $0.89 \pm 0.18$ & $1.03 \pm 0.08$ & 0.2411 \\
\hline Lysine & $1.02 \pm 0.10$ & $0.95 \pm 0.16$ & $1.01 \pm 0.08$ & 0.6919 \\
\hline Methanol & $1.08 \pm 0.48$ & $1.01 \pm 0.31$ & $0.94 \pm 0.22$ & 0.8168 \\
\hline Methionine & $1.10 \pm 0.16$ & $1.06 \pm 0.24$ & $1.07 \pm 0.11$ & 0.8907 \\
\hline Methylsuccinate & $1.01 \pm 0.15$ & $1.13 \pm 0.37$ & $0.99 \pm 0.22$ & 0.5316 \\
\hline Ornithine & $0.89 \pm 0.11$ & $0.87 \pm 0.26$ & $1.01 \pm 0.16$ & 0.0749 \\
\hline Phenylalanine & $1.03 \pm 0.11$ & $0.95 \pm 0.18$ & $1.01 \pm 0.12$ & 0.4840 \\
\hline Proline & $0.97 \pm 0.18$ & $0.98 \pm 0.15$ & $1.03 \pm 0.17$ & 0.6228 \\
\hline Propylene glycol & $1.20 \pm 0.94$ & $1.41 \pm 0.94$ & $1.53 \pm 0.60$ & 0.9182 \\
\hline Serine & $0.94 \pm 0.17$ & $1.00 \pm 0.19$ & $1.07 \pm 0.12$ & 0.1717 \\
\hline Threonine & $1.16 \pm 0.43$ & $1.04 \pm 0.16$ & $1.04 \pm 0.07$ & 0.9140 \\
\hline Tyrosine & $1.04 \pm 0.11$ & $0.95 \pm 0.14$ & $1.00 \pm 0.12$ & 0.4469 \\
\hline Valine & $0.99 \pm 0.08$ & $0.84 \pm 0.37$ & $1.03 \pm 0.08$ & 0.4337 \\
\hline
\end{tabular}

Values as mean \pm standard deviation. 
Figure 1. Fold-change (Post/Pre) of the six metabolites that presented significant changes. High-intensity resistance exercise (HI-RE), low-load resistance training with blood flow restriction (LL-BFR), control (CON). " Significantly different from CON; ${ }^{\dagger}$ Significantly different from LL-BFR. Data are mean \pm SD. 

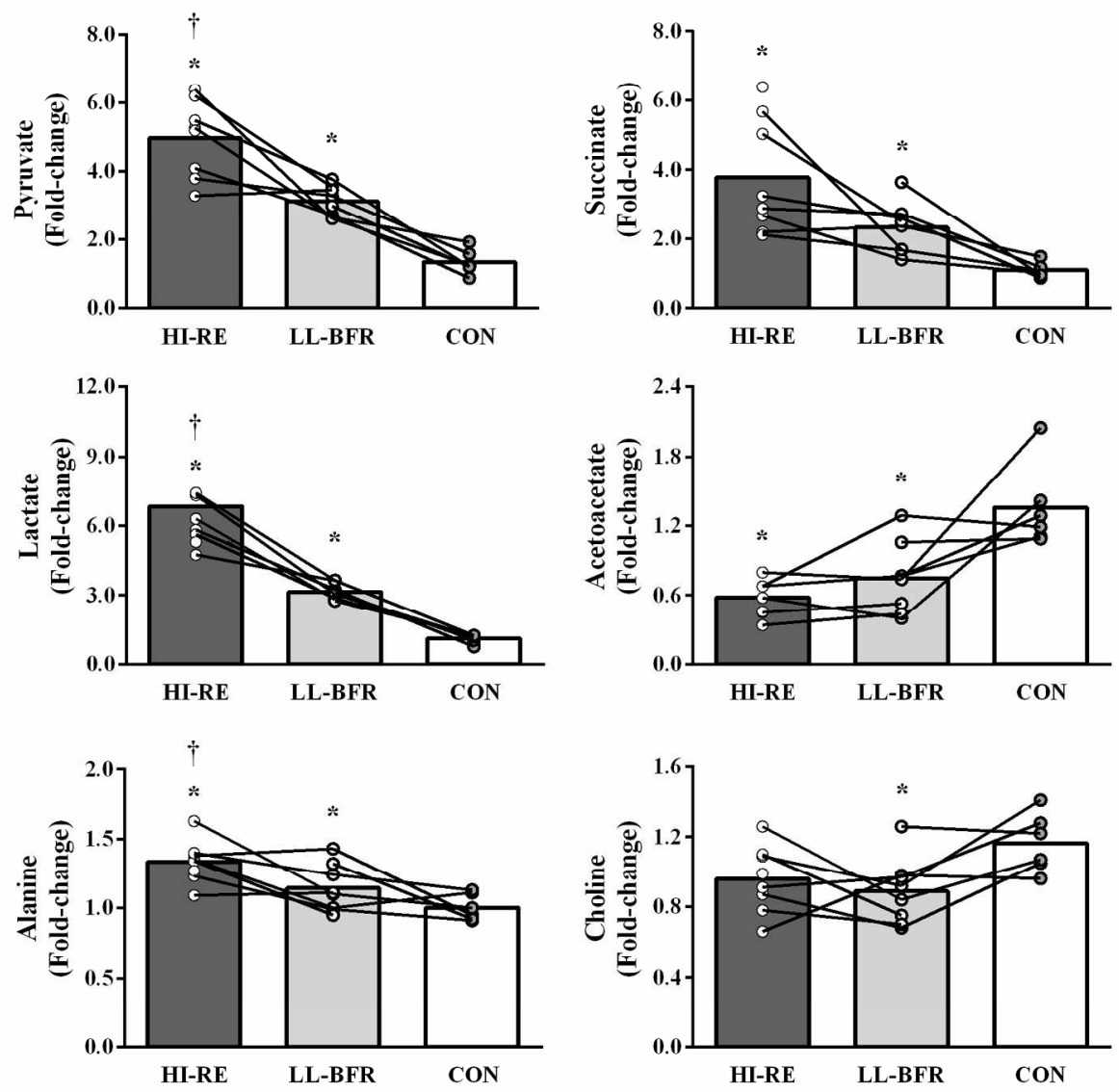

Figure 1. Fold-change (Post/Pre) of the six metabolites that presented significant changes. High-intensity resistance exercise (HI-RE), low-load resistance training with blood flow restriction (LL-BFR), control condition (CON). *Significantly different from CON; + Significantly different from LL-BFR. Data are mean \pm SD.

$176 \times 166 \mathrm{~mm}(300 \times 300 \mathrm{DPI})$ 